\title{
Spatially resolved heat conduction in polar perhydrotriphenylene inclusion compounds studied by means of thermal waves
}

\author{
M. Wübbenhorst ${ }^{\text {a) }}$ and J. van Turnhout \\ Department of Polymer Technology, Delft University of Technology, P.O. Box 5045, \\ 2600 GA Delft, The Netherlands \\ A. Quintel and J. Hulliger b) \\ Department of Chemistry and Biochemistry, University of Berne, Freiestr. 3, 3012 Berne, Switzerland
}

(Received 23 March 2000; accepted for publication 17 May 2000)

\begin{abstract}
Scanning pyroelectric microscopy was applied to study the local thermal diffusivity in polar perhydrotriphenylene (PHTP) inclusion compounds. A thermal wave technique with special heat flow and pyroelectric detection principles was used to measure the longitudinal $\left(K_{\|}\right)$and transversal $\left(K_{\perp}\right)$ thermal diffusivity of needle shaped crystals. As expected, we have found a higher thermal conductivity along the channel direction: $K_{\|}>K_{\perp} . K_{\|}$also showed a variation along the needle axis, the $K_{\|}$values near the capping faces being higher than in the center region. Based on these observations we suggest a relation between the longitudinal thermal conductivity and the local density of orientational defects of the dipolar guest molecules which reaches a maximum in the area around the seed. These findings are in agreement with previous pyroelectric studies, revealing a macrodomain structure and a spatially dependent pyroelectric activity for two different PHTP inclusion compounds. C 2000 American Institute of Physics. [S0021-8979(00)05416-5]
\end{abstract}

\section{INTRODUCTION}

In channel-type inclusion compounds of perhydrotriphenylene (PHTP) various guest molecules can be aligned into chains within the parallel channels. ${ }^{1}$ In case of the inclusion of push-pull type $A-\pi-D$ guest molecules ( $A$ : electronic acceptor substituent, $D$ : electronic donor substituent, $\pi$ : conjugated spacer), long polar chains are formed in the individual channels. The polar ordering originates from selective interactions of the terminal $A$ and $D$ groups, leading to a bipolar macroscopic state, where cone-like macrodomains build up a stationary polarization inside the two cones. ${ }^{2}$ The inner regions of the cones show a higher density of orientational defects, which should influence the thermal conductivity of the material.

Recently, ${ }^{2-5}$ we have shown that the pyroelectric effect allows one to study the polar domain structure of PHTP inclusion compounds by means of scanning pyroelectric microscopy (SPEM). A prerequisite for a proper analysis of SPEM results is the knowledge of the thermal properties of the crystals, since the effective local volume wherein the pyroelectric response is generated, strongly depends on the thermal diffusivity $K$ and the radial frequency of the temperature variation $\omega$, which both determine the thermal diffusion length $\mu$, according to $\mu=\sqrt{2 K / \omega}$. It seems likely that, in return, one can acquire information about the thermal properties, provided that the polarization profile parallel to the heat flow direction is known.

In the present article we will demonstrate the capability of SPEM for determining the local thermal diffusivity of PHTP inclusion compounds. Experiments using SPEM will

\footnotetext{
${ }^{a}$ Electronic mail: wuebbenhorst@ $@$ tnw.tudelft.nl

b)Electronic mail: juerg.hulliger@iac.unibe.ch
}

be described, which allow the assessment of both the transversal $(\perp)$ and longitudinal $(\|)$ component of the thermal diffusivity. Results will be discussed in the framework of orientational disorder of the guest molecules (NPP: 1-(4nitrophenyl)piperazine, INBP: 4-iodo-4'-nitrobiphenyl) in PHTP inclusion compounds.

\section{PYROELECTRIC CALORIMETRY: BASIC SCHEMES AND RELATIONS}

\section{A. Detection of the temperature distribution by means of the pyroelectric effect}

The measurement of dynamic thermal properties like specific heat $c$ and thermal diffusivity $K=k / c \rho$ ( $k$ $=$ thermal conductivity, $\rho=$ density) generally requires the generation and detection of well-defined time-dependent temperature changes within the sample. Many experimental techniques for determining $c \rho$ and $K$ apply continuous thermal waves, ${ }^{6}$ i.e., they use a sinusoidally modulated heat flux resulting in a periodic time and position dependent temperature distribution $T_{\sim}(x, y, z, t)$. A measurement of the temperature profile (or parts of it) by an appropriate detection technique then yields the thermal properties, provided the proper model is used.

Thermal wave techniques utilizing the pyroelectric effect for the measurement of the thermal diffusivity $K$, are well established for thin films or rod-like samples, which typically show one-dimensional heat transport. The analytical solution of the corresponding heat transport equation is given in Appendix A.

Let us now discuss three particular configurations for thermal wave experiments based on pyroelectric detection shown in Fig. 1. 
a

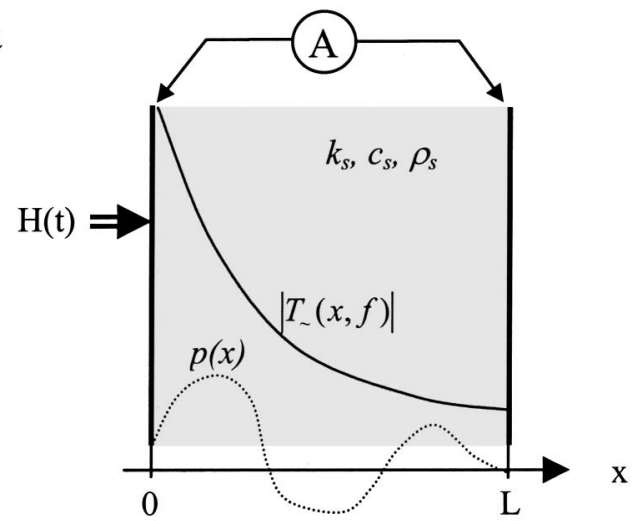

Response of pyroelectric sample:

b

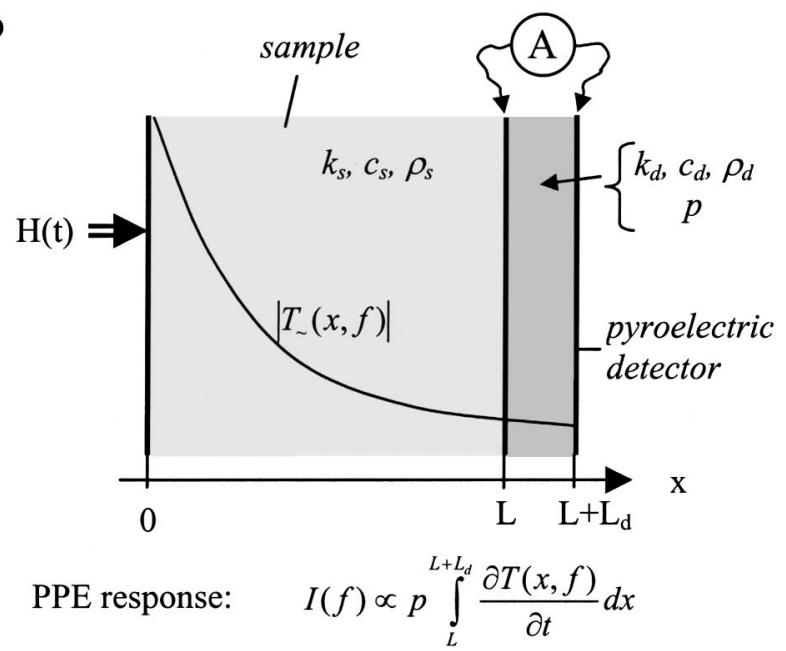

c

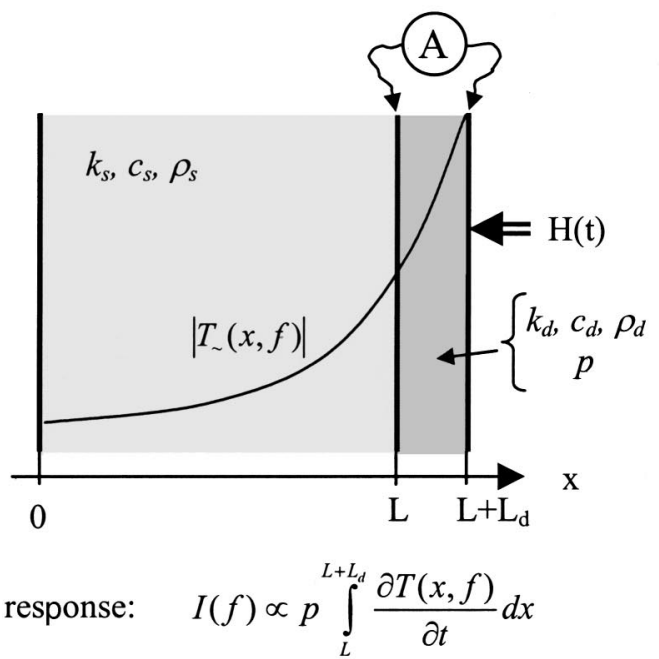

FIG. 1. Three basic configurations for the measurement of (1D) thermal properties of a dielectric layer using the pyroelectric effect: (a) pyroelectrically active sample with known pyroelectric distribution, (b) pyroelectric calorimeter in PPE mode and, (c) IPPE-mode.

Pyroelectricity is a property of dielectric materials, which show a temperature-dependent, macroscopic (permanent or spontaneous) polarization $P$, i.e., they generate surface charges as a result of a temperature change $\Delta T(t)$.
These charges can either be detected directly or as a pyroelectric current $I(t)$. Assuming a flat pyroelectric layer according to Fig. 1(a) (covered with two electrodes at $x=0$ and $x=L)$ the pyroelectric current density $j(t)$ is given by

$$
j(t)=p \frac{d\langle T\rangle}{d t},
$$

where $p$ denotes the pyroelectric coefficient at constant pressure, $p=d P / d T .^{7}$ Note that Eq. (1) only holds for homogeneously polarized materials. In order to account for a spatially inhomogeneous polarization $P(x)$, knowledge of the particular one-dimensional temperature distribution $T(x, t)$ is required. For a periodic time dependence $T(x, \omega)$ the amplitude of the complex pyroelectric current $I_{\sim}$ can be written as $^{8}$

$$
\begin{aligned}
I_{\sim}(\omega)= & \left(\alpha_{p}+\alpha_{x}-\alpha_{\epsilon}\right) \frac{A}{L} \int_{0}^{L} P^{*}(x) \frac{\partial T(x, \omega)}{\partial t} d x \\
& +\alpha_{p}\langle P\rangle_{x} A \frac{d\langle T\rangle_{x}}{d t},
\end{aligned}
$$

where $\alpha_{p}, \alpha_{x}$, and $\alpha_{\epsilon}$ are the temperature coefficient of the polarization $\left(\alpha_{p}\right)$, the linear expansion coefficient $\left(\alpha_{x}\right)$, and the temperature coefficient $\left(\alpha_{\epsilon}\right)$ of the permittivity $\epsilon$, respectively. $A$ is the heated area, and $L$ the thickness of the sample. Note that the overall polarization distribution has been decomposed into its mean value $\langle P\rangle_{x}$ and the remaining nonhomogeneous part $P^{*}(x)$. In other words, the classical pyroelectric coefficient $p$ of a homogeneous pyroelectric material is represented by the second term in Eq. (2), i.e., $p=\alpha_{p}\langle P\rangle_{x}$.

From previous work we know $^{2-5}$ that in PHTP inclusion compounds, the mean polarization (when averaged over the entire volume) vanishes, therefore, the second term can be neglected. The remaining first term represents the deviation of the polarization from its mean value. In contrast to homogeneous pyroelectric samples, two additional material properties have to be taken into account: $\alpha_{x}$ and $\alpha_{\epsilon}$.

Up to now we have implicitly assumed that the length over which heat will be transported coincides with the electrically active length as indicated in Eq. (1) by the interval of integration $0 \leqslant x \leqslant L$. The generated pyroelectric response will then depend on unknown properties such as the polarization distribution and the thermal diffusivity $K$. The common strategy of measuring $K$ is therefore the separation of the thermal transport region from the layer wherein the amplitude and phase of the thermal waves are detected. This technique is often called pyroelectric calorimetry, which exists in several options. ${ }^{6,9-11}$ In its simplest form, a pyroelectric calorimeter consists of two layers, (i) one acting as pyroelectric detector with known thermal properties and a homogeneous pyroelectric activity $p$, and (ii) a layer with unknown thermal properties as sample.

The two possible positions of the sample layer with respect to the heat source provide two operation modes for a pyroelectric calorimeter, (i) photopyroelectric (PPE), ${ }^{12}$ whereby the sample layer is exposed to the heat source [Fig. 1(b)], and (ii) photopyroelectric (IPPE) ${ }^{9}$ inverse detection [Fig. 1(c)]. 


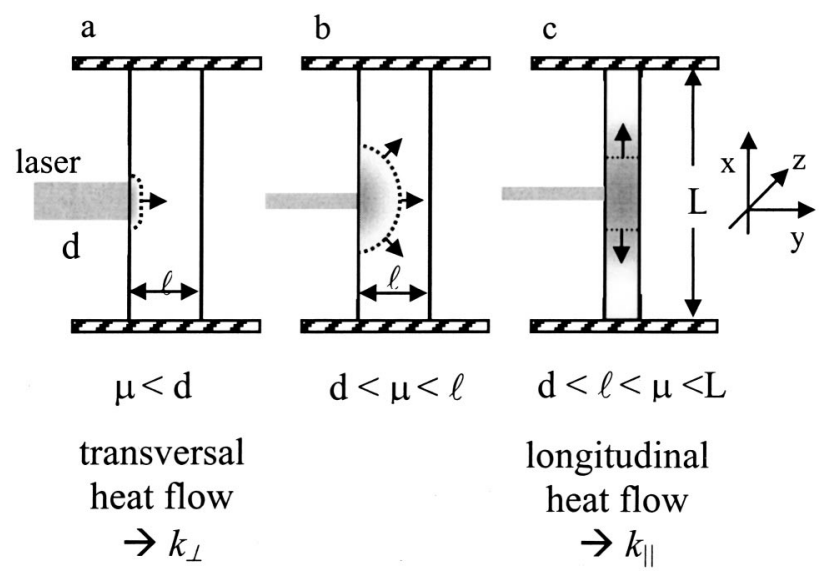

FIG. 2. Three special cases of heat flow in a rod-like sample: (a) flat transversal heat flow, (b) semispherical, and (c) longitudinal heat flow.

Both the PPE and IPPE modes require a particular geometry for the detector, which matches the shape of the sample in order to ensure good thermal contact. This requirement can easily be fulfilled in flat thin-layer calorimeters. However, it is hard to realize for small needle-like samples. One attractive way to circumvent the complications of a separate pyroelectric sensor is the use of the pyroelectric properties of the sample. Here, we have used the pyroelectric inhomogeneities measured by SPEM in order to probe the thermal diffusivity.

\section{B. Heat transport approximations for rod-like samples}

The three-dimensional heat flow in a rod-like crystal has been discussed by Quintel et al. ${ }^{3}$ in connection with the interpretation of SPEM. Considering a sample (thickness $L$, diameter $l$ ) which absorbs laser radiation (beam diameter $d$ ) at the surface, one can distinguish three cases of simplified heat transport as shown in Fig. 2, each occurring in a characteristic range of frequencies and thus thermal diffusion lengths:

(a) $\mu<d$ (high frequencies, $10^{2}-10^{5} \mathrm{~Hz}$ ): Onedimensional (1D) heat flow perpendicular to the needle direction (transversal heat flow). In this situation the heat propagation is controlled by the transversal thermal diffusivity $K_{\perp}$.

(b) $\quad d<\mu<l$ (medium frequencies, $10 \mathrm{~Hz}$ ): semispherical $\left(K_{\perp}=K_{\|}\right)$or semiellipsoidal $\left(K_{\perp} \neq K_{\|}\right)$heat flow.

(c) $l<\mu<L$ (low frequencies, $<1 \mathrm{~Hz}$ ): One-dimensional longitudinal heat flow parallel to the needle direction determined by $K_{\|}$. We assume that the heat source is delocalised over a length region $\Delta L \approx l$. This approximation holds for crystals with a large aspect ratio.

It is obvious that only case (a) enables a thermal resolution better than or equal to the far field optical resolution, which is the normal operation mode of surface-SPEM. ${ }^{3,5}$ For the purpose of measuring thermal transport properties, simple 1D-temperature distributions given by (a) and (c) are preferred, since they depend only on $K_{\perp}$ or $K_{\|}$.

\section{EXPERIMENTAL SETUP AND MATERIALS}

\section{A. Experimental setup}

A schematic drawing of the equipment is shown in Fig. 3. A laser diode (LISA HL25/M2, $5 \mathrm{~mW}, \lambda=635 \mathrm{~nm}$ ) was used as an intensity modulated radiation source, whose beam was guided and focused by an optical microscope onto the needle-shaped pyroelectric sample. An additional lens near the microscope side port ensured the coincidence of the laser focus plane with that of the main optical path, which allowed inspection of the sample's adjustment during handling and measurement.

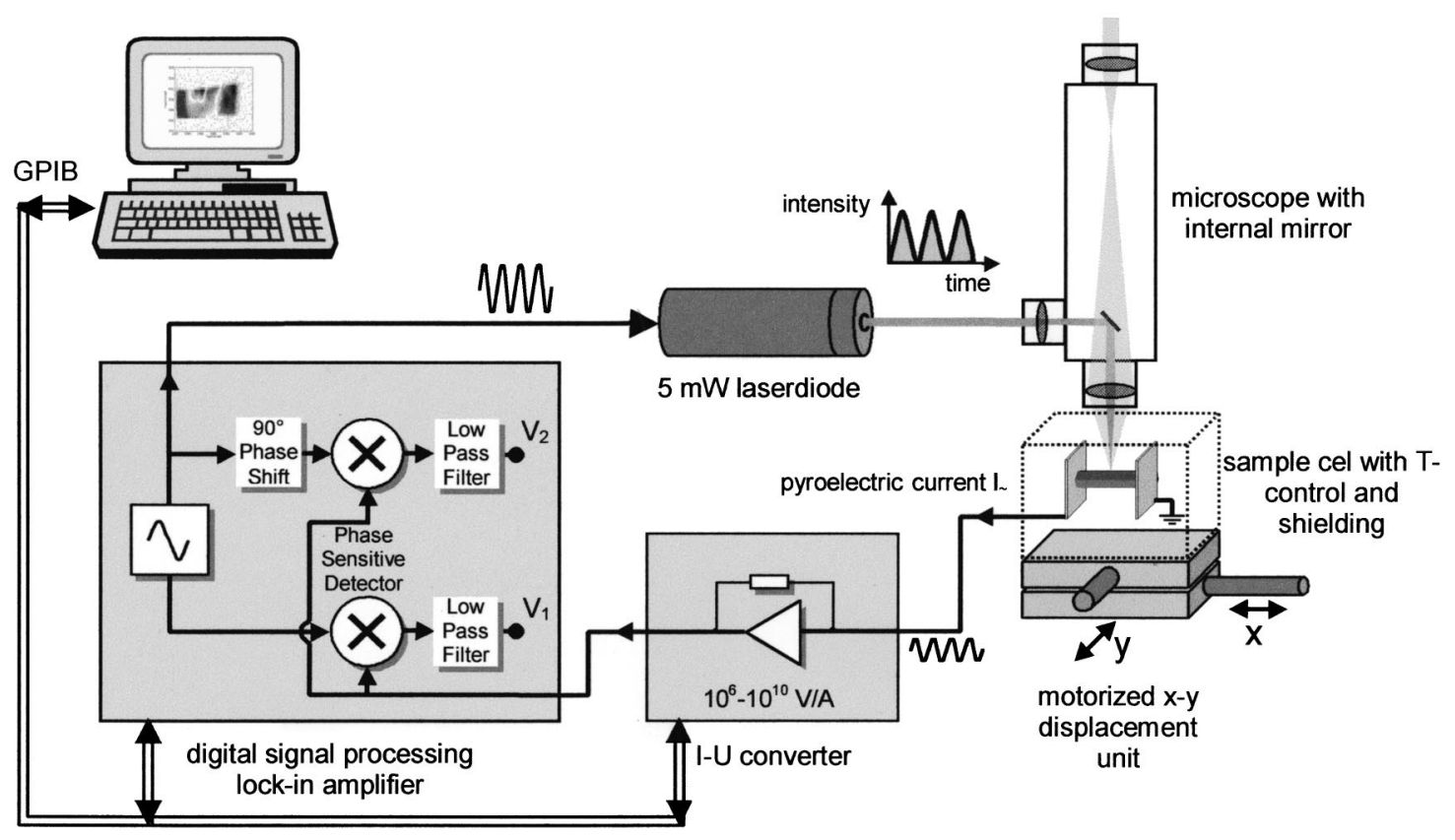

FIG. 3. Experimental setup of SPEM. 
The sample was placed between two plate electrodes (area: $1 \mathrm{~cm}^{2}$ ), glued with conducting silver paste on the grounded electrode, thermally and mechanically separated by an air gap (width $<100 \mu \mathrm{m}$ ) from the other electrode. Twodimensional scanning of the sample was performed by moving the whole sample cell by means of a motorized $x-y$ displacement unit. Since the smallest diameter of the focused laser spot was about $10 \mu \mathrm{m}$, a typical scan increment of 20 $\mu \mathrm{m}$ along the $x$ and $y$ axes was used. For effective absorption of the laser radiation, all samples were blackened by dipping into an ethanol solution of a black dye.

The pyroelectric current was converted into voltage by a low noise/high gain current amplifier (Keithley Model 428, gain typically $10^{8}-10^{9} \mathrm{~V} / \mathrm{A}$ ), which ensured dynamic "short-circuit" conditions. Further amplification and phasesensitive analysis of the pyroelectric response took place in a digital signal processing lock-in amplifier (Stanford Research 850) using the internal since generator as a reference and driving voltage for the modulation of the laser diode at frequencies from $0.1 \mathrm{~Hz}$ up to $10 \mathrm{kHz}$. All measurements were performed under ambient conditions, at $25^{\circ} \mathrm{C}$.

\section{B. Materials}

Inclusion crystals containing two different $A-D$ disubstituted chromophores were investigated: PHTP-NPP crystals [NPP: 1-(4-nitrophenyl)piperazine] and PHTP-INBP crystals [INBP: 4-iodo-4'-nitrobiphenyl]. Needle-shaped crystals of $1-3 \mathrm{~mm}$ in length and $0.2-0.5 \mathrm{~mm}$ in thickness were obtained by crystallization from the solution, resulting in crystals with a nearly hexagonal cross section. The needle axis corresponds to the channel axis (denoted by $x$, in Fig. 2). Further details concerning the crystal growth and the sample preparation are given in Ref. 3.

\section{EXPERIMENTAL RESULTS}

\section{A. Transversal thermal diffusivity in PHTP-INBP inclusion compounds}

We have already mentioned, that the interpretation of the frequency dependence of the pyroelectric response $I_{\sim}(\omega)$, generated by a pyroelectric distribution $p(x)$ and/or the thermal diffusivity $K$, requires a (one-dimensional) heat flux parallel to $p(x)$.

The probing of $K_{\perp}$ requires a well defined polarization profile $p(y)$ perpendicular to the long axis of the crystal. In this respect, PHTP inclusion compounds only show typical polarization reversals in the long direction rather than suitable transversal polarization inhomogeneities. ${ }^{2,5}$

In a few cases however, crystals with a complex domain structure were obtained, providing more than one polarization inversion. Such peculiarities result from irregularities during the growth process. This may include incorporation of smaller crystals into a larger one. One prominent example of an irregular PHTP-INBP crystal is given in Fig. 4 showing a three-dimensional representation of its pyroelectric surface activity. ${ }^{3}$

The SPEM images taken from this crystal clearly reveal the existence of a flat domain in the top layer of the sample with a polarization direction opposite to that of the neighbor-

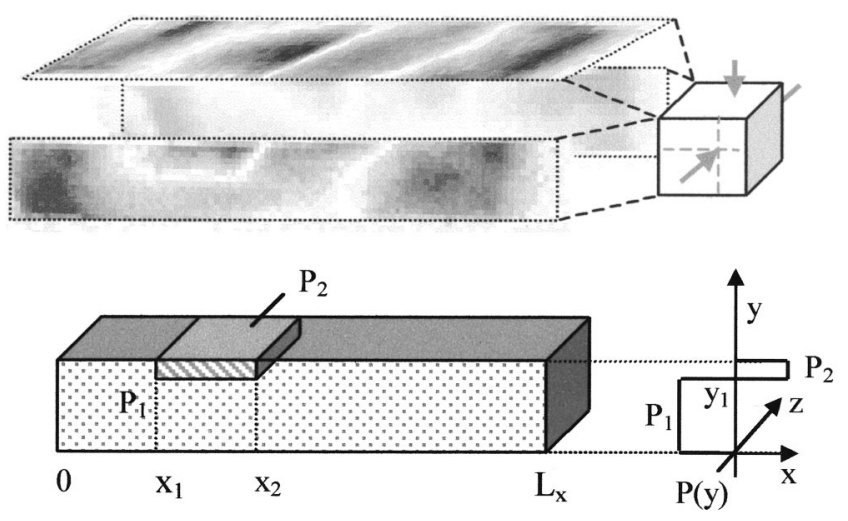

FIG. 4. Perspective view in three dimensions of the pyroelectric surface activity of a PHTP-INBP crystal.

ing regions. Additional SPEM experiments were made at lower frequencies probing the average pyroelectric activity across the entire cross section (Fig. 6 in Ref. 3). These measurements substantiated the assumption of a flat pyroelectric domain as indicated in Fig. 4.

This pyroelectric anomaly was studied in detail, since it represents a simple and sharp lateral polarization profile. Pyroelectric spectra were taken from $1 \mathrm{~Hz}$ up to $30 \mathrm{kHz}$ using a defocused laser spot $(d \sim 200 \mu \mathrm{m})$, which illuminated the crystal region to probe either from the bottom $(y=0)$ or from the top $(y=L)$ side. The corresponding pyroelectric spectra $I_{0}(f)$ and $I_{L}(f)$ are shown in Fig. 5.

For the analysis of the pyroelectric spectra we assume:

The transversal pyroelectric profile $p(y)$ can be modeled by a block profile described by only two free parameters, viz. (i) the position of the polarization reversal, and (ii) the pyroelectric coefficient $p_{1}$ in the layer $0<y<L_{l}$.

(ii) A transversal pyroelectric profile $p(y)$ creates essentially the same pyroelectric spectra when sensed perpendicular to the $y$ axis by electrodes at the end faces of the crystal as an equivalent distribution $p(x)$, sensed in the conventional measuring configuration illustrated in Fig. 1(a), provided that the heat flow is transversal, i.e., parallel to the $p(y)$ profile.

The validity of approximation (i) concerning the block-like profile is supported by the sharp domain boundaries visible in the pyroelectric image in Fig. 4. Within the two domains of opposite polarity, roughly the same value of the polarization is likely, according to the growth model recently developed by Hulliger et al. ${ }^{1}$

Assumption (ii) leads us back to the fundamentals of the pyroelectric response equation [Eq. (2)] derived by Lang. ${ }^{8}$ In his derivation Lang has formally shown that two different contributions to the pyroelectric response exist, which refer to the homogeneous polarization part (mean value $\langle P\rangle$ ) and to the nonhomogeneous part $P^{*}$. This can be rationalized in the following way: A homogeneous polarization [layer thickness $L$, see Fig. 6(a)], can be fully described by its equivalent (virtual) surface charges $\sigma_{P 0}=P$ and $\sigma_{\mathrm{PL}}=-P$ at the respective positions $x=0$ and $x=L$. When the polar material 
a)

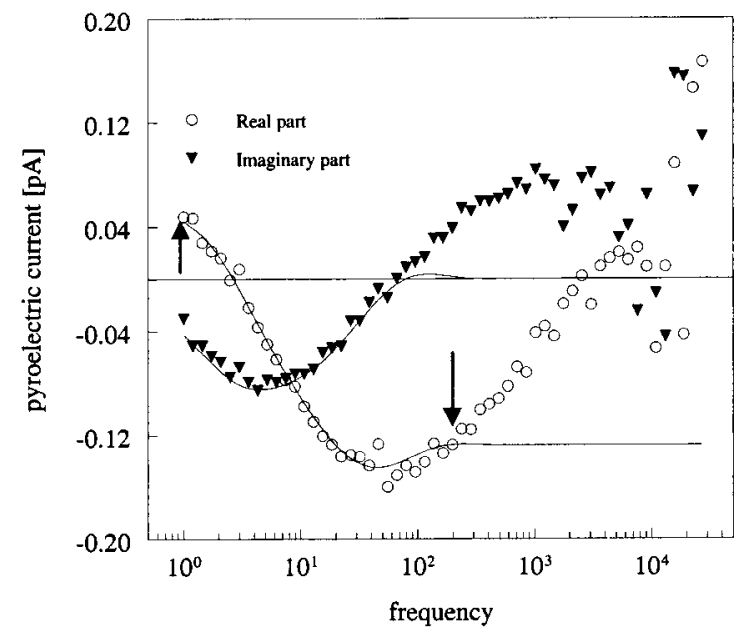

b)

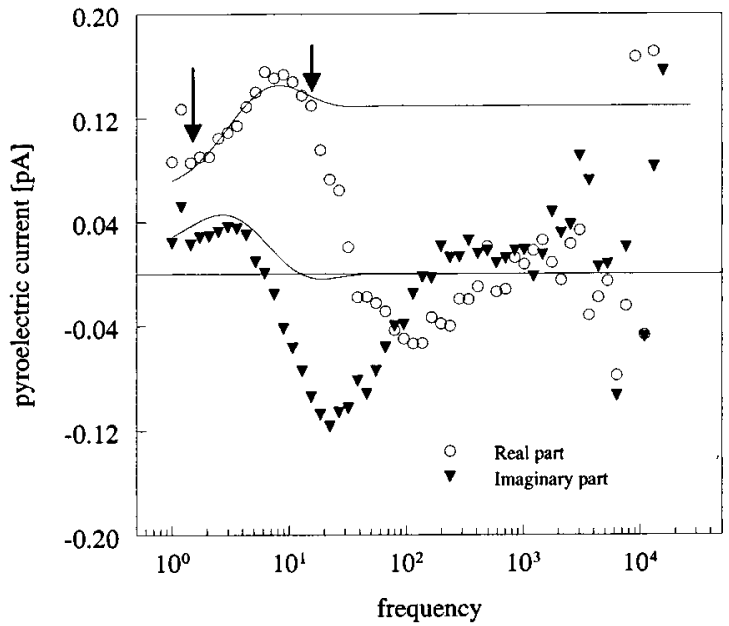

FIG. 5. Measured (symbols) and fitted (lines) pyroelectric spectra of a PHTP-INBP crystal taken at $y=0$ (top) and $y=L_{y}$ (bottom). The arrows indicate the frequency range in which the data were fitted. Note that only $\operatorname{Re}\{I\}$ data were used for the NLLS fit.

is short circuited by electrodes, these virtual charges will be compensated by real charges at the electrodes, leading to a zero electric field inside and outside the sample. Changing the temperature by $\Delta T$ will now change the polarization, which results in a corresponding change in the (detectable) surface charge density according to $\Delta \sigma_{P 0}=-\Delta \sigma_{\mathrm{PL}}=\Delta P$ $=p \Delta T$. Consequently, thickness changes and changes in the permittivity, quantified by $\alpha_{x}$ and $\alpha_{\epsilon}$, play no significant role.

In contrast, a polarization distribution with zero mean value according to Fig. 6(b) possesses polarization charges in the bulk of the sample, which create an image charge variation at the surface. The latter not only depends on the polarization itself but also on the relative position of $\sigma_{P 0}$ and $\sigma_{\mathrm{PL}}$ including the (temperature dependent) profile of the dielectric constant $\epsilon$. As a consequence, the quantities $\alpha_{x}$ and $\alpha_{\epsilon}$ have to be considered.

Let us discuss now the situation presented in Fig. 6(c), which combines a longitudinal with a transversal polarization distribution. For simplicity we assume a polar domain of the cross section $L_{y} \times L_{z}$ and length $d=x_{2}-x_{1}$, whereby the polarization only varies in the $y$ direction. Full irradiation of

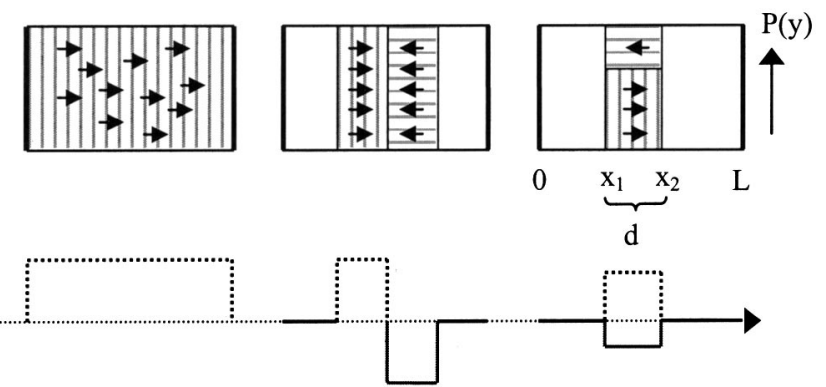

FIG. 6. Three basic polarization distributions in a dielectric layer with a polarization direction $\|$ to the main direction $x$ : (a) homogeneous polarization, (b) longitudinal profile $P(x)$, and (c) transversal profile $P(y)$ within a slab.

the sample at $y=0$ over the surface area $L_{z} \times d$ ensures onedimensional transversal heat flow in the $y$ direction at sufficiently short thermal diffusion lengths.

In order to take the transversal polarization distribution $P(y)$ into account, we consider small slices $L_{x} \times d y \times L_{z}$ which at the position $y$ contribute to the pyroelectric current by $d I \sim$

$$
\begin{aligned}
d I_{\sim}(y, \omega)= & \left(\alpha_{p}+\alpha_{x}-\alpha_{\epsilon}\right) \frac{d y L_{z}}{L_{x}} \\
& \times \int_{0}^{L_{x}} P^{*}(x, y) \frac{\partial T(x, \omega)}{\partial t} d x \\
& +\alpha_{p} d y L_{z}\langle P(x, y)\rangle_{x} \frac{d\langle T(x, y, \omega)\rangle_{x}}{d t} .
\end{aligned}
$$

Inserting the particular temperature distribution $T_{0}(y)$ and polarization profile $P(y)$ (noting that $T_{0}(y), P(y)$ are constant between $x_{1}$ and $x_{2}$ ) one can solve the integral over $x$

$$
\begin{aligned}
d I_{\sim}(y, \omega)= & d \frac{L_{z}}{L_{x}} \frac{d T_{0}(y, \omega)}{d t}\left\{\left(\alpha_{p}+\alpha_{x}-\alpha_{\epsilon}\right) P^{*}(y)\right. \\
& \left.+\alpha_{p}\langle P(x, y)\rangle_{x}\right\} d y .
\end{aligned}
$$

Integration over $y$ results in an expression for the total pyroelectric current

$$
\begin{aligned}
I_{\sim}(\omega)= & d \frac{L_{z}}{L_{x}} \int_{0}^{L_{y}}\left[\left(\alpha_{p}+\alpha_{x}-\alpha_{\epsilon}\right) P^{*}(y)\right. \\
& \left.+\alpha_{p}\langle P(x, y)\rangle_{x}\right] \frac{d T_{0}(y, \omega)}{d t} d y .
\end{aligned}
$$

As expected, the first term of Eq. (5) solely derives from the transversal polarization profile $P(y)$ in analogy to Eq. (2). The second part, however, depends on both the longitudinal and transversal polarization distribution and will be small owing to the averaging over the $x$ direction. In the special case of zero polarization outside the heated volume, the term $\langle P(x, y)\rangle_{x}$ is proportional to $P(y)$.

It should be mentioned that for the derivation of Eq. (5) the thermoelastic and thus the piezoelectric contributions were neglected, i.e., the generation of mechanical stress profiles due to inhomogeneous thermal expansion in the sample is excluded. In particular, steep temperature profiles present 
TABLE I. Results for $k_{\perp}, K_{\perp}$ and $y_{I}$ from NLLS fit to the pyroelectric spectra given in Fig. 5.

\begin{tabular}{|c|c|c|}
\hline & $I_{0}$ spectrum & $I_{L}$ spectrum \\
\hline$k_{\perp}(\mathrm{W} / \mathrm{mK})$ & $0.11 \pm 0.05$ & $0.12 \pm 0.03$ \\
\hline$K_{\perp}\left(\mathrm{m}^{2} / \mathrm{s}\right)$ & $7.8 \times 10^{-8}$ & $8.3 \times 10^{-8}$ \\
\hline Position of polarization reversal & $y_{I}=(144 \pm 9) \mu \mathrm{m}$ & $\begin{array}{l}L-y_{I}=(52 \pm 10) \mu \mathrm{m} \\
\Rightarrow y_{I}=(148 \pm 10) \mu \mathrm{m}\end{array}$ \\
\hline
\end{tabular}

at high modulation frequencies may result in mechanical stresses near the surface region close to the heat source. Although these thermoelastic properties may be too complex to be taken into account, one can expect only specific deviations in the pyroelectric current spectrum at high frequencies, whereas at sufficiently low frequencies no internal stresses are present.

The pyroelectric spectra given in Fig. 5 were analyzed by a nonlinear least-squares (NLLS) fit based on the wellknown Marquardt-Levenberg algorithm. This fit procedure minimizes the sum of squared differences between the experimental data $\operatorname{Re}\left\{I^{\exp }\right\}$ and the values calculated by the nonlinear pyroelectric response equation $\operatorname{Re}\left\{I^{\text {calc }}\right\}$

$$
\sum_{i}\left(\operatorname{Re}\left\{I_{i}^{\exp }\right\}-\operatorname{Re}\left\{I_{i}^{\text {calc }}\right\}\right)^{2} \rightarrow \operatorname{Min} .
$$

The actual function $I^{\text {calc }}$ was obtained by combining Eq. (5) (first term) with the temperature distribution $T_{\sim}(x, \omega)$ according to Eq. (A2) and a polarization block profile $P(y)$. Taking e.g. $P_{1}=1$ and $P_{2}=-1$ we arrive at an expression for the complex pyroelectric current

$$
\begin{aligned}
I_{\sim}(\omega)= & C\left\{P_{1} \int_{0}^{y_{1}} \frac{d T_{0}(y, \omega)}{d t} d y\right. \\
& \left.+P_{2} \int_{y_{1}}^{L_{y}} \frac{d T_{0}(y, \omega)}{d t} d y\right\}
\end{aligned}
$$

with

$$
\begin{aligned}
\frac{d T_{0}(y, \omega)}{d t}= & \frac{i \omega H_{0} L_{y}}{k D^{*}} \\
& \times \frac{\sinh \frac{D^{*} y}{L_{y}}+\cosh D^{*} \sinh D^{*}\left(1-\frac{y}{L_{y}}\right)}{\sinh ^{2}\left(D^{*}\right)},
\end{aligned}
$$

where $D^{*}=L_{y}(1+i) / \mu$. Since we are not interested in the absolute pyroelectric coefficient of the sample, all constants present in Eq. (5) as well as the sign of the block-like polarization profile are represented by an effective parameter $C$. This reduces the set of parameters to six, which include three known and three adjustable quantities according to

$$
I(\omega)=f\left(C, c_{p} \rho, L_{y}, y_{1}, k, \omega\right) .
$$

Using $L_{y}=200 \mu \mathrm{m}$ and $c \rho=1.5 \times 10^{6} \mathrm{~J} / \mathrm{m}^{3} \mathrm{~K}^{3}$ we obtained stable fit parameters for $k$ and $y_{1}$, which are listed in Table I. The calculated spectra $\operatorname{Re}\left\{I^{\text {calc }}\right\}$ are shown in Fig. 5 as lines together with the experimental data. For the fits, only the real part of the pyroelectric data was used since the imaginary

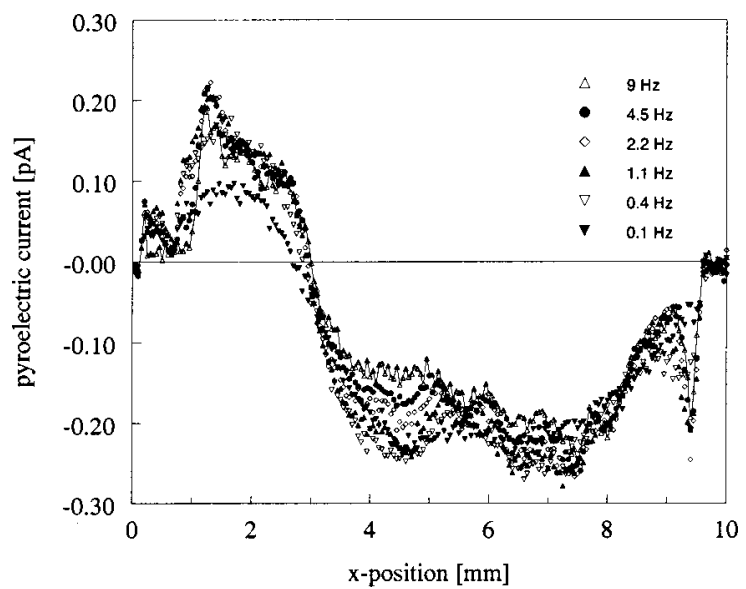

FIG. 7. Pyroelectric $x$ scans $\operatorname{Re}\{I(x)\}$ of a large PHTP-NPP inclusion crystal at different frequencies.

and real part of the pyroelectric spectra are interrelated, i.e., they obey the Kramers-Kronig relations if the data are consistent. For this reason we have also given the measured and calculated imaginary current component in Fig. 5, which demonstrate a good agreement even in the imaginary spectra and thus prove the reliability of the real part spectra.

Inspecting the fit results in Table I we find a surprisingly good agreement between the thermal conductivity and position of the polarization reversal $y_{1}$ determined by the two independent pyroelectric experiments. Obviously, all simplifications concerning the pyroelectric block profile, the thermal model, and the pyroelectric current generation are reasonable. At higher frequencies (see Fig. 5) the simple model function [Eq. (7)] failed as is manifested by an increasing deviation of $\operatorname{Re}\left\{I^{\exp }\right\}$ from the high frequency plateau predicted by Eq. (7). Consequently, these high frequency data have not been considered in the fit procedure. Most likely, the high frequency decrease of $\operatorname{Re}\left\{I^{\exp }\right\}$ is caused by less polar surface regions and/or the increased influence of mechanical stresses at high frequencies as suggested earlier.

\section{B. Longitudinal thermal conductivity of a PHTP-NPP inclusion crystal}

Figure 7 shows the influence of the modulation frequency on pyroelectric scans in the length direction of a PHTP-NPP inclusion crystal. As expected, the spatial dependence of the pyroelectric current changes from a sharp and detailed curve at high frequencies to a smooth curve at very low frequencies. The reason is twofold: On the one hand, the direction of the heat flow changes from a transversal to a finally longitudinal one [case(a) $\rightarrow$ case(c) in Fig. 2]. On the other hand, at sufficiently low frequencies at which longitudinal heat transport is established, the increasing thermal diffusion length $\mu$ leads to increasing delocalization of the heated region accompanied by averaging of the pyroelectric signal over a larger sample volume.

Clearly, this averaging effect becomes more pronounced with lower frequency. It depends directly on the local thermal diffusivity responsible for the heat delocalization. Since the pyroelectric $x$ scan contains information about the pyroelectric profile in the length direction $p(x)$ as well as on the 
spatially dependent thermal diffusivity $K(x)$, it seems attractive to analyze at a scan position $x_{0}$ the $I\left(x_{0}, \omega\right)$ dependence given by

$$
\begin{aligned}
I_{\sim}\left(x_{0}, \omega\right)= & \frac{A}{L_{x}}\left(\alpha_{p}+\alpha_{x}-\alpha_{\epsilon}\right) \\
& \times \int_{0}^{L_{x}} P\left(x^{\prime}\right) \frac{d T\left(x_{0}, x^{\prime}, \omega\right)}{d t} d x^{\prime},
\end{aligned}
$$

where $x_{0}$ and $x^{\prime}$ denote the position of the heat source and the integration variable, respectively. Equation (10) was obtained from Eq. (2), again by neglecting the second term. For short thermal diffusion lengths the polarization profile $P\left(x_{0}\right)$ is closely approximated by the pyroelectric $x$ scan $I\left(x_{0}\right)$ itself during to the displacement of the heat source. In the case of low frequencies (large thermal diffusion lengths) however, the thermal properties show up. This leads us to a strategy of finding the $K(x)$ distribution.

From the data presented in Fig. 7 we have selected the curve at $f=1.1 \mathrm{~Hz}$ which still shows sufficient details implying not much thermal smoothing yet. The approximation is now made that $I\left(f_{1}=1.1 \mathrm{~Hz}, x_{0}\right)$ is proportional to $P\left(x_{0}\right)$. The function $K\left(x_{0}\right)$ can then be found by minimization of

$$
\begin{gathered}
\sum_{i=1}^{N}\left[I_{i}^{\exp }\left(f_{2}, x_{i}\right)-C_{a} \sum_{j=1}^{N} I_{j}^{\exp }\left(f_{1}, x_{j}^{\prime}\right)\right. \\
\left.\times \frac{d T\left(f_{2}, x_{i}, x_{j}^{\prime}, K\left(x_{j}^{\prime}\right)\right.}{d t}\right]^{2} \rightarrow \text { Min },
\end{gathered}
$$

in which $C_{a}$ is a constant and $I_{i}^{\exp }$ and $I_{j}^{\exp }$ denote the real part of the measured pyroelectric current at the frequency $f$ whereby the condition $f_{2}<f_{1}$ must be fulfilled. Equation (11) can be solved, in principle, by the same NLLS technique as described above, yielding the constant $C_{a}$ and the unknown distribution function $K(x)$. However, in order to obtain the general solution $K(x)$, the proper thermal model should be used which allows for a spatially dependent thermal diffusivity $K(x)$. Since such a general temperature distribution $T[x, K(x)]$ can only be obtained by numerical techniques, we assume $K\left(x_{0}\right)=$ constant within the temperature profile generated around a given position $x_{0}$ of the heat source. With respect to Eq. (11), this means that the distribution $K\left(x_{j}^{\prime}\right)$ should be replaced by $K\left(x_{i}\right)$, i.e., near each scan position $x_{i}$ the sample is regarded as thermally homogeneous.

As a consequence, $K(x)$ varies much less than the polarization profile, which seems a reasonable assumption. In order to solve Eq. (11), we need the solution of the appropriate thermal model for a heat source at an adjustable scan position $x_{0}$. A simple way to derive the expression $T\left(x_{0}, x^{\prime}, \omega\right)$ uses the idea that a thermally homogeneous layer which contains a heat source at position $x_{0}$, can be regarded as a combination of two single layers each characterized by their own temperature distribution $T_{L}\left(x_{0}, x^{\prime}\right.$ $\left.\leqslant x_{0}\right)$ and $T_{R}\left(x_{0}, x^{\prime} \geqslant x_{0}\right)$ according to Eqs. (A2) and (B5)(B7) (see Appendices A and B). Some calculated tempera-

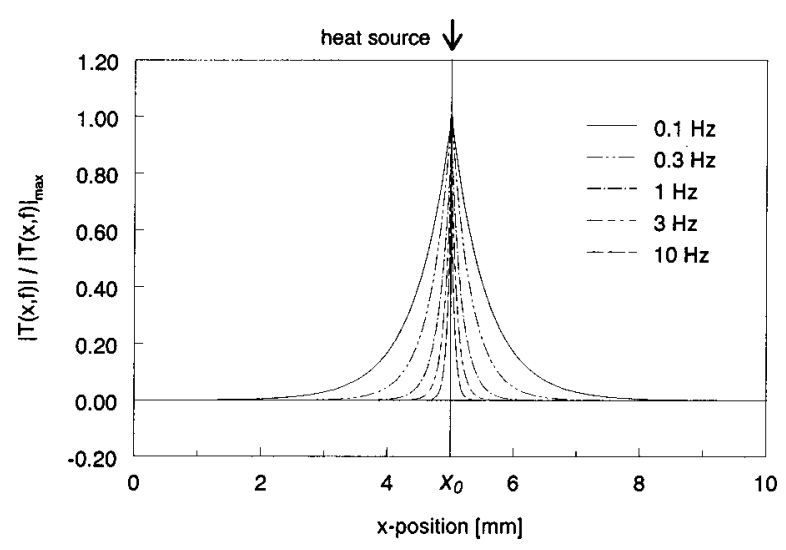

FIG. 8. Normalized amplitude of the temperature variation $T(x, \omega)$ at scan position $x_{0}$ for different frequencies calculated using Eqs. (A2), (B1), and (B2). Thermal parameters are: $K=10^{-7} \mathrm{~m}^{2} / \mathrm{s}, c \rho=2.3 \times 10^{6} \mathrm{~J} / \mathrm{m}^{3} \mathrm{~K}$.

ture profiles $T(x, \omega)$ as a function of the frequency are given in Fig. 8. Note that the profiles will be symmetric except near the ends of the sample.

Equation (11) can now be solved using the temperature distribution at each position of the heat source and assuming a trial function for $K(x)$. We used a block-like function $K_{i}$, $i=1 \ldots 10$ which divides the sample length in ten intervals and allows one to adjust the thermal diffusivity in each interval separately. Although this procedure causes some discontinuities in the calculated $I\left(x_{0}\right)$ dependence, such a discrete $K\left(x_{0}\right)$ representation enables us to account for abrupt changes of the heat flow as, e.g., present at the crystal end faces as can be seen in Fig. 9 in the intervals on the far left and far right. Finer spacing of the intervals would certainly improve the $K\left(x_{0}\right)$ resolution but would also increase the processing time for the NLLS-fit drastically. ${ }^{13}$

\section{DISCUSSION}

The experimental findings can be summarized as follows.

(1) The lateral thermal diffusivity of a PHTP-INBP inclusion crystal shows a $K_{\perp}$ value of $8 \times 10^{-8} \mathrm{~m}^{2} / \mathrm{s}$, which is in the range typical of amorphous and (nonoriented) semicrystalline polymers.

(2) The longitudinal thermal conductivity $K_{\|}$of an inclusion crystal varies by almost one order of magnitude, from $5 \times 10^{-8} \mathrm{~m}^{2} / \mathrm{s}$ up to $6 \times 10^{-7} \mathrm{~m}^{2} / \mathrm{s}$. Interestingly, remarkably high $K_{\|}$values are found in the outer regions of crystals, while $K_{\|}$is lowest near the nucleation region, i.e., in the part of polarization reversal.

In other words, the thermal anisotropy is most pronounced in regions of higher orientational order of the guest molecules (large net polarization).

A link between the thermal conductivity and the molecular order can be made using the simple Debye equation ${ }^{14}$

$k=\frac{1}{3} \rho c v \bar{l}$,

where $\rho$ is the density, $c$ the mass specific heat at constant pressure, $v$ the sound velocity, and $\bar{l}$ the mean-free path of 

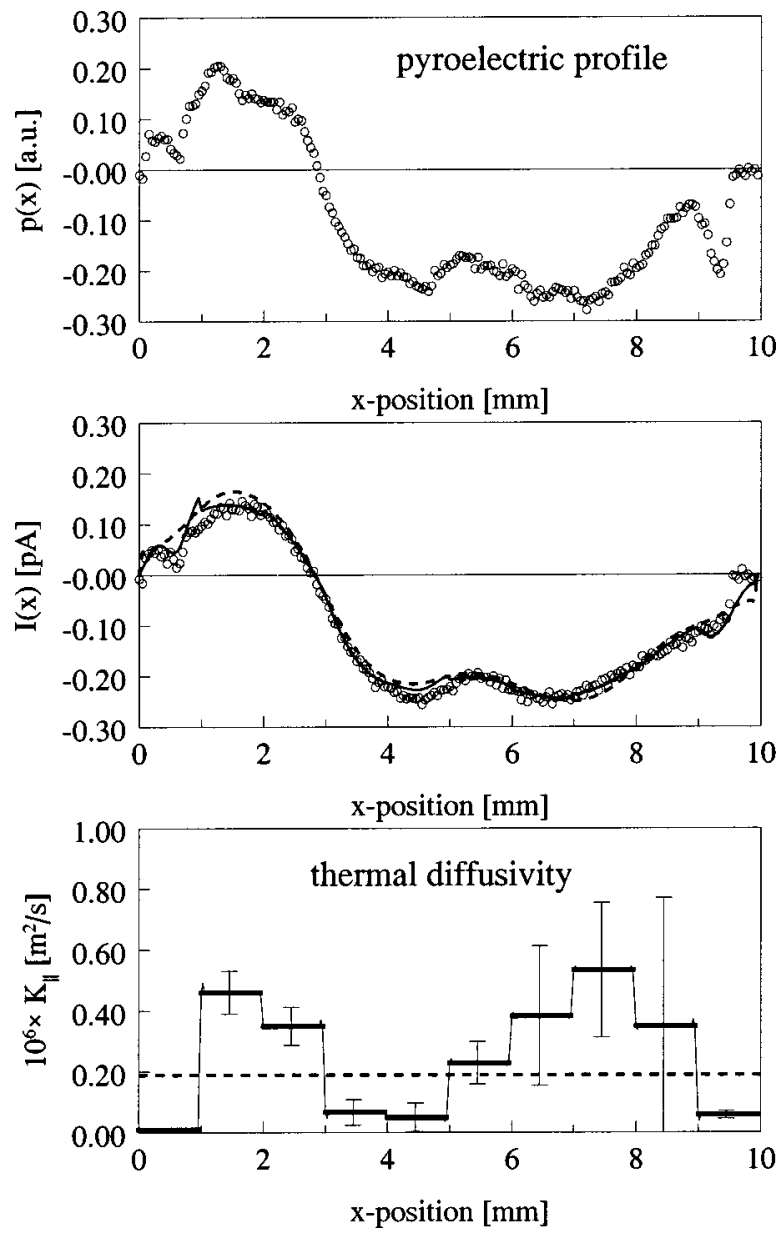

FIG. 9. Approximated polarization profile (top), smoothed polarization profile (center), and calculated distribution $K_{\|}(x)$ obtained for a PHTP-NPP single crystal.

(acoustical) phonons. Among these four parameters, the density and specific heat are not very sensitive to the molecular structure and the morphology of organic solids and show only a weak temperature dependence around room temperature. Consequently, the thermal anisotropy must originate from either an anisotropic sound velocity or anisotropy in the phonon mean-free path. Studies on highly oriented polyethylene have shown that both properties may contribute to thermal anisotropy.

It is obvious that in PHTP inclusion crystals both the host lattice and the one dimensionally arranged guest molecules could give rise to thermal anisotropy. However, there is not much data in the literature on thermal conduction in organic structures based on aligned rod-like molecules. Few results have been reported about thermal diffusion measurements on liquid crystals. ${ }^{15,16}$ The thermal properties of nematic liquid crystals may be comparable to some extent with channel-type inclusion compounds. For example, Caerels et al. ${ }^{15}$ have measured the thermal conductivity $k_{\|}$of nematic liquid crystals along the long axis (\| to the nematic director). A maximum value of $K_{\|}=0.28 \mathrm{~W} / \mathrm{mK}$ as well as a thermal anisotropy of $K_{\|} / K_{\perp}>2$ could be explained by the high aspect ratio of the rod-like molecules. Similar results were obtained by Akahane et al. ${ }^{16}$ who studied the thermal diffusivity $K_{\|}$of a smectic liquid crystal. Again, the thermal diffusivity $K_{\|}=1.35 \times 10^{-7} \mathrm{~m}^{2} / \mathrm{s}$ and thermal anisotropy $K_{\|} / K_{\perp} \sim 2$ were discussed in terms of molecular anisotropy accompanied with high molecular ordering as present in smectic A phases.

Following the idea that the phonon mean-free path length should be in the order of the dimensions of the molecular building blocks, one might expect a lateral mean-free path length $\bar{l}_{\perp}$ close to the interchannel distance of $15 \AA$. Using $v=2300 \mathrm{~m} / \mathrm{s}$ (typical sound velocity of a semicrystalline polymer), $K_{\perp} \approx 8 \times 10^{-8} \mathrm{~m}^{2} / \mathrm{s}$ and the relation $\bar{l}_{\perp}$ $=3 K_{\perp} / v$ we would obtain $\bar{l}_{\perp}=1.0 \AA$, this is unrealistically low.

In order to account for the high and strongly varying longitudinal thermal conductivity $K_{\|}$, we will consider two contributions to the thermal conductivity, $K_{\| h}$ (host) and $K_{\| g}$ (guest). Assuming that phonon transport along the PHTP stacks and the H-bonded guest molecules can be regarded as independent processes, we can express the overall thermal diffusivity according to a parallel model by

$$
K_{\|}=\chi K_{\| g}+(1-\chi) K_{\| h},
$$

where $\chi$ corresponds to the concentration of guest molecules of about $10 \%$. Supposing that $K_{\| h}$ does not depend either on the ordering of the guest molecule chains or on the position, we attribute the lowest measured values of $K_{\|}$to the host lattice, i.e., $K_{\| \min } \cong K_{\| h}=5 \times 10^{-8} \mathrm{~m}^{2} / \mathrm{s}$. When inserting this value in Eq. (13) together with the maximum thermal conductivity found in Fig. $9\left(K_{\| \max }=6 \times 10^{-7} \mathrm{~m}^{2} / \mathrm{s}\right)$ we obtain a thermal diffusivity contribution of guest molecular chains of $K_{\| g}=5.6 \times 10^{-6} \mathrm{~m}^{2} / \mathrm{s}$. Using Eq. (12), this results in a phonon free-path length of $\bar{l}_{\| g}=72 \AA$. This much higher value may be put into relation to one-dimensional phonon transport within hydrogen bonded, highly ordered $A-\pi-D \cdots A-\pi-D$ sequences along the PHTP channels. Knowing the NPP-repetition length of $12 \AA$, we may conclude that the average distance between $\mathrm{A}-\mathrm{A}$ or $\mathrm{D}-\mathrm{D}$ defects is of the order of five guest molecules. These defectfree lengths are 4 orders of magnitude shorter than the corresponding lengths predicted by the Markov theory of 1D growth. ${ }^{5,17}$ Although x-ray analysis of the PHTP-NPP crystal structure ${ }^{18}$ led to a lower limit of ordered lengths, this value is still 2 orders of magnitude higher than the phonon free-path length just calculated. Consequently, the phonon free-path length is far too low to justify our former assumption, that the longitudinal thermal conductivity is directly related to the orientation defect density within single guest chains. In other words, there is no experimental evidence for one-dimensional phonon transport along "thermally isolated" guest molecule chains, which could give rise for enormously high $\bar{l}_{\| g}$ values. Obviously, there is no sufficient vibrational decoupling between guest and host sublattice, which actually form a commensurate lattice in the case of PHTP-NPP. More likely, long-range 2D translational disorder, i.e., promoted by orientational disorder of neighboring dipole chains, provides a possible link between $K_{\|}$and the local net polarization, and explains the limited $K_{\|}$value. 


\section{SUMMARY AND CONCLUSIONS}

The anisotropic thermal diffusivity of polar perhydrotriphenylene (PHTP) inclusion crystals was studied by means of a versatile technique, scanning pyroelectric microscopy (SPEM). For this purpose, SPEM was used in two operation modes: First, the (local) surface pyroelectric activity was measured in the scanning mode in order to guess the three-dimensional pyroelectric distribution $p(x, y, z)$, and second, pyroelectric spectra were taken using a fixed, large area thermal excitation with a defocused laser source (spectrum mode). By combining the pyroelectric response obtained at a fixed frequency and moving heat source with that obtained at variable frequency and fixed/moving heat source we could determine both the transversal $\left(K_{\perp}\right)$ and longitudinal $\left(K_{\|}\right)$thermal diffusivity of needle-shaped inclusion crystals.

Since our approach is based on pyroelectric inhomogeneities in the heat flow direction of interest, samples with a specific domain structure had to be found, which could be modeled by either a transversal or a longitudinal pyroelectric profile $p(y)$ or $p(x)$. Although this seems to be a strong limitation, we have demonstrated for PHTP-based inclusion compounds, that such desired pyroelectric domain types exist, caused by arbitrary defects during crystallization or due to polar growth into twinned domains. In particular, two large, needle-shaped inclusion crystals were investigated, each representing a particular one-dimensional polarization profile: (1) a PHTP-INBP crystal, showing a well defined bipolar transversal polarization distribution, and (2) a PHTP-NPP sample, characterized by a sharp pyroelectric domain reversal along the crystal needle axis.

Of these samples, the thermal diffusivities $K_{\perp}$ and $K_{\|}$ were measured by applying the appropriate one-dimensional heat flow parallel to the direction of the polarization variation. Transversal heat flow was induced by illuminating the sample with a fixed large laser spot, resulting in pyroelectric frequency spectra $I_{\sim}[p(y), f] . K_{\perp}$ was then obtained by fitting of $I_{\sim}(f)$ to the proper pyroelectric profile assuming a one-dimensional thermal model.

In the longitudinal case, the pyroelectric response was acquired at different (low) frequencies and at different positions (line scan) along the longitudinal pyroelectric profile $p(x)$. It could be shown, that this procedure even enabled us to reveal the spatial dependence of the longitudinal thermal diffusivity $\left[K_{\|}(x)\right]$.

As expected for channel-type materials, a higher thermal conductivity was found along the channel direction (growth direction): $K_{\|}>K_{\perp} . K_{\|}$appeared to vary along the crystal length showing higher $K_{\|}$values near the capping faces than in the center region where the nucleation started. These observations strongly suggest a relation between the experimentally found phonon mean-free path length and orientational defects of the polar guest molecules. The latter show a maximum in the region of the seed.

\section{APPENDIX A: ONE-DIMENSIONAL TEMPERATURE} DISTRIBUTION IN A SOLID SAMPLE

The propagation of heat in a thermally conducting solid can be described by Fourier's law (1) (one-dimensional case). Introducing a characteristic parameter $K$, the thermal diffusivity, we have

$$
\frac{\partial T}{\partial t}=K \frac{\partial^{2} T}{\partial x^{2}} .
$$

To obtain particular solutions $T(x, t)$, Eq. (A1) must be solved for a defined geometry and initial boundary conditions. ${ }^{19}$ We will restrict our calculation to the solution of the common thermal one-layer model depicted in Fig. 1(a).

A thin layer (thickness $L$ ) is assumed to be thermally insulated against its environment (no heat losses); the heat source is located at $x=0$ which corresponds to the case of absorbing radiation power $H(t)=H_{0}[1+\cos (\omega t)]$ at the sample surface. According to Ref. 19 the periodical term $T_{\sim}(x, \omega)$ of the solution of Eq. (A1) reads

$$
T_{\sim}(x, \omega)=\frac{H_{0} L}{k D^{*}} \frac{\sinh D^{*} \frac{x}{L}+\cosh D^{*} \sinh D^{*}\left(1-\frac{x}{L}\right)}{\sinh ^{2}\left(D^{*}\right)},
$$

with $\mu=(2 K / \omega)^{1 / 2}$ and $D^{*}=L(1+i) / \mu$. Note that $T_{\sim}(x, \omega)$ represents the amplitude of the complex temperature. Its time dependence can be obtained by multiplying the right hand side of Eq. (A2) with $\exp (i \omega t)$.

From Eq. (A2) we notice some important properties of $T(x, \omega)$ under the condition of $\mu \ll L$ (semi-infinite limit).

(1) The amplitude of the temperature variation follows an exponential decay characterized by the thermal diffusion length $\mu$.

(2) $\mu$ depends only on $K$ and can be controlled by the angular modulation frequency $\omega$.

(3) The propagation of temperature variations shows the characteristics of a strongly damped wave (thermal wave) which wavelength $\lambda=2 \pi \mu$ and a constant attenuation of $|T(\lambda, \omega)| /|T(0, \omega)|=e^{-2 \pi} \cong 0.00187$.

\section{APPENDIX B: TEMPERATURE DISTRIBUTION IN A 1D HEAT CONDUCTING SOLID WITH A HEAT SOURCE POSITIONED AT $x=x_{0}$-SIMPLE CALCULATION}

We envisage that the sample can be split at the scanning position $x_{0}$ in a left and right part. We first assume that the full sinusoidal heat flux $H_{0}$ is supplied to either the left or the right layer. Then we can readily calculate the temperature profiles in these layers $T_{\mathrm{LS}}\left(x, \omega, H_{0}\right)$ and $T_{\mathrm{RS}}\left(x, \omega, H_{0}\right)$ with Eq. (A2). In practice, the heat flux $H_{0}$ will be split at $x_{0}$ in a flux to the left and to the right, $H_{0 L}$ and $H_{0 R}$. This will result in two modified temperature profiles $T_{L}\left(x, \omega, H_{0 L}\right)$ and $T_{R}\left(x, \omega, H_{0 R}\right)$, which are related to $T_{\mathrm{LS}}\left(x, \omega, H_{0}\right)$ and $T_{\mathrm{RS}}\left(x, \omega, H_{0}\right){ }^{20}$

This simple relation can be easily derived from the conservation of energy at $x_{0}$ and the matching of the amplitude and phase of the temperatures at $x_{0^{-}}$and $x_{0^{+}}$, which give 


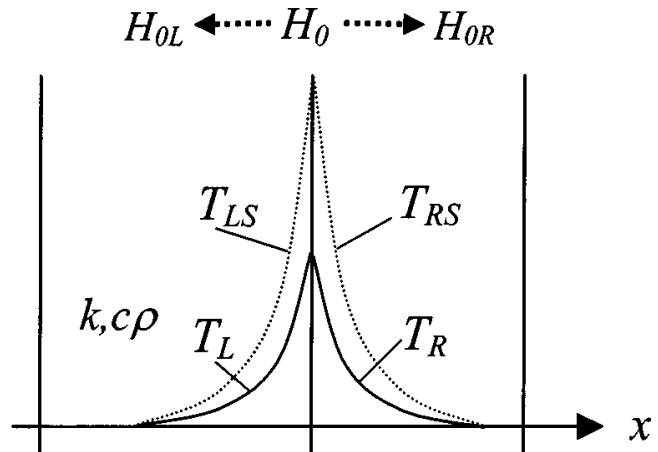

$$
x_{0}-L_{1} \quad x_{0} \quad x_{0}+L_{2}
$$

FIG. 10. Illustration of the temperature profiles generated by the laser source at position $x_{0}$ during an $x$ scan. In order to simplify the calculations the sample is virtually split in a left and right layer.

$$
\begin{aligned}
& H_{0}=H_{0 L}+H_{0 R}, \\
& T_{L}\left(x_{0}, \omega, H_{0 L}\right)=T_{R}\left(x_{0}, \omega, H_{0 R}\right) .
\end{aligned}
$$

Writing for the ratio between $H_{0 L}$ and $H_{0 R}{ }^{21}$

$$
\alpha=\frac{H_{0 L}}{H_{0 R}}
$$

we have from Eq. (B1)

$$
H_{0 L}=\frac{\alpha}{1+\alpha} H_{0} \text { and } H_{0 R}=\frac{H_{0}}{1+\alpha} .
$$

Because of the linear dependence of $T\left(x, \omega, H_{0}\right)$ on $H_{0}$ according to Eq. (A2), we can therefore write

$$
\begin{aligned}
& \begin{aligned}
T_{L}\left(x, \omega, H_{0 L}\right) & =T_{L}\left(x, \omega, \frac{\alpha H_{0}}{1+\alpha}\right) \\
= & \frac{\alpha}{1+\alpha} T_{\mathrm{LS}}\left(x, \omega, H_{0}\right), \quad x \leqslant x_{0} \\
\text { and } T_{R}\left(x, \omega, H_{0 R}\right) & =T_{R}\left(x, \omega, \frac{H_{0}}{1+\alpha}\right) \\
& =\frac{1}{1+\alpha} T_{\mathrm{RS}}\left(x, \omega, H_{0}\right), \quad x \geqslant x_{0} .
\end{aligned}
\end{aligned}
$$

By substituting Eq. (B2) in Eqs. (B5) and (B6) we get for $\alpha$

$$
\alpha=\frac{T_{\mathrm{RS}}\left(x_{0}, \omega, H_{0}\right)}{T_{\mathrm{LS}}\left(x_{0}, \omega, H_{0}\right)} .
$$

Note that except near the ends $\alpha$ will equal 1 , so that for the most part $T_{L}$ and $T_{R}$ will be the same (see Fig. 10). Further note that this simple procedure only holds for the case of identical thermal properties in the left and right layer, at least near the scanning point $x_{0}$. This is also the reason why we took a block-like profile for $K(x)$.

${ }^{1}$ J. Hulliger, P. J. Langley, O. König, S. W. Roth, A. Quintel, and P. Rechsteiner, Pure Appl. Opt. 7, 221 (1998).

${ }^{2}$ J. Hulliger, A. Quintel, M. Wübbenhorst, P. J. Langley, S. W. Roth, and P. Rechsteiner, Opt. Mater. 9, 259 (1998).

${ }^{3}$ A. Quintel, J. Hulliger, and M. Wübbenhorst, J. Phys. Chem. B 102, 4277 (1998).

${ }^{4}$ S. W. Roth, P. J. Langley, A. Quintel, M. Wübbenhorst, P. Rechsteiner, P. Rogin, O. König, and J. Hulliger, Adv. Mater. 10, 1543 (1998).

${ }^{5}$ A. Quintel, S. W. Roth, J. Hulliger, and M. Wübbenhorst, Mol. Cryst. Liq. Cryst. 338, 243 (2000).

${ }^{6} \mathrm{H}$. Coufal, in New Characterization Techniques for Thin Polymer Films, edited by H. M. Tong and L. T. Nguyen (Wiley, New York, 1990), p. 231-264.

${ }^{7}$ Landolt-Börnstein, New Series Group III: Crystals and Solid State Physics Vol. 11, edited by K.-H. Hellwege (Springer, Berlin, 1979).

${ }^{8}$ S. B. Lang, Ferroelectrics 118, 343 (1991).

${ }^{9}$ M. Chirtoc, V. Tosa, D. Bicanic, and P. Toefs, Ber. Bunsenges. Phys. Chem. 95, 766 (1991).

${ }^{10}$ M. Wübbenhorst, J. van Turnhout, and L. Alili, Ferroelectrics 165, 153 (1995).

${ }^{11}$ G. J. Klap, M. Wübbenhorst, J. van Turnhout, J. C. Jansen, and H. van Bekkum, Prog. Surf. Sci. 105, 2093 (1997).

${ }^{12}$ M. Chirtoc and G. Mihailescu, Phys. Rev. B 40, 9606 (1989).

${ }^{13}$ The NLLS-fit was done by a TURBO PASCAL based program. The typical execution time was several hours on a Pentium II PC, using 200 measurement points, 10 intervals, and 10 iterations.

${ }^{14}$ C. Kittel, Introduction to Solid State Physics (Wiley, New York, 1996).

${ }^{15}$ J. Caerels, E. Schoubs, and J. Thoen, Liq. Cryst. 22, 659 (1997).

${ }^{16}$ T. Akahane, M. Kondoh, K. Hashimoto, and M. Nakagawa, Jpn. J. Appl. Phys., Part 1 26, 1000 (1987).

${ }^{17}$ A. Quintel and J. Hulliger, Chem. Phys. Lett. 312, 567 (1999).

${ }^{18}$ O. König, H.-B. Bürgi, T. Armbruster, J. Hulliger, and T. Weber, J. Am. Chem. Soc. 119, 10632 (1997).

${ }^{19}$ H. S. Carslaw and J. C. Jaeger, Conduction of Heat in Solids, 2nd ed. (Clarendon, Oxford, 1959).

${ }^{20} \mathrm{~F}$. Marlow (personal communication, 1994).

${ }^{21}$ Since the temperatures are complex in the $\omega$ domain, we should allow $H_{0 L}$ and $H_{0 R}$ to be complex as well. The same applies to $\alpha$. 